\title{
Ventilação mecânica não invasiva na bronquiolite viral aguda: estudo de coorte retrospectivo
}

\author{
Non-invasive mechanical ventilation in acute viral bronchiolitis: retrospective cohort study
}

\author{
Aline Morás Borges ${ }^{a}$, Camila Wohlgemuth Schaan ${ }^{b}$, Sergio Luis Amantéac, Janice Luisa Lukrafka ${ }^{d}$ \\ a Fisioterapeuta. Especialização em Terapia Intensiva no formato de Residência Multiprofissional pela Universidade Federal de Ciências da Saúde de Porto Alegre (UFCSPA) e \\ Irmandade Santa Casa de Misericórdia de Porto Alegre (ISCMPA). Fisioterapeuta do Hospital da Criança Santo Antônio (ISCMPA). \\ b Fisioterapeuta. Mestre em Ciências das Saúde (Cardiologia) pelo Instituto de Cardiologia do Rio Grande do Sul (IC). Fisioterapeuta do Hospital de Clínicas de Porto Alegre (HCPA). \\ c Médico. Doutor em Ciências Pneumológicas pela Universidade Federal do Rio Grande do Sul (UFRGS). Professor adjunto da UFCSPA. \\ d Fisioterapeuta. Doutora em Ciências Médicas pela UFRGS. Professora adjunta da UFCSPA.
}

RESUMO

Objetivo: Avaliar a utilização da ventilação mecânica não invasiva (VMNI) sobre parâmetros clínicos nas primeiras 24 horas em pacientes internados em uma emergência pediátrica com diagnóstico de bronquiolite viral aguda (BVA).

Materiais e Métodos: Estudo de coorte retrospectivo através de coleta de dados de prontuários de crianças internadas com diagnóstico de BVA que fizeram uso de VMNI em uma emergência pediátrica durante dois invernos consecutivos. Dados coletado: demográficos (sexo, idade), características clínicas (frequência cardíaca, frequência respiratória, saturação periférica de oxigênio), tempo de uso da VMNI, internação em UTI pediátrica e tempo de internação. Foram utilizados testes de Friedman e Wilcoxon para comparação de variáveis clínicas entre cada período de tempo, teste de Mann-Withney e ANOVA para comparações entre o grupo que utilizou e não utilizou VMNI.

Resultados: Amostra composta por 14 pacientes, o tipo de vírus mais prevalente foi o vírus sincicial respiratório, o modo ventilatório não invasivo predominantemente utilizado foi o CPAP. Não houve diferença significativa dos sinais vitais em relação ao momento da instalação da VMNI, seis, 12 e 24 horas. Na utilização de oxigênio suplementar houve diferença significativa entre o momento da instalação da VMNI e após 24 horas. Quando comparadas as características clínicas dos pacientes que falharam na $\mathrm{VMNI}$ e necessitaram de suporte ventilatório invasivo, a frequência cardíaca diferiu significativamente tanto no momento da instalação da VMNI como após 6 horas.

Conclusão: Houve redução significativa da necessidade de oxigênio suplementar após 24 horas da instalação da VMNI. Não foram observadas diferenças significativas nas demais características clínicas dos pacientes.

Palavras-chave: bronquiolite viral; ventilação não invasiva; pediatria. hospitalized in a pediatric emergency with diagnosis of acute viral bronchiolitis (AVB).

Materials and Methods: A retrospective cohort study including medical records of children hospitalized with a diagnosis of AVB who used NIV in the pediatric emergency for two consecutive winters. Collected data: demographic (sex, age), clinical characteristics (heart rate, respiratory rate, peripheral oxygen saturation), time of use of NIV, hospitalization in pediatric ICU and length of hospital stay. Friedman and Wilcoxon tests were used to compare clinical variables between each time period, Mann-Withney test and ANOVA for comparisons between the group that used and did not use NIV.

Results: The sample consisted of 14 patients, the most prevalent type of virus was respiratory syncytial virus (RSV), and the noninvasive ventilation mode predominantly used was CPAP. There was no significant difference in vital signs in relation to the time of installation of NIV, six, 12 and 24 hours. There was no significant difference in the use of supplemental oxygen between the time of installation of NIV and after 24 hours. When comparing the clinical characteristics of patients who failed NIV and required invasive ventilation, heart rate differed significantly both at the time of installation of NIV and after 6 hours.

Conclusion: There was a significant reduction in the need for supplemental oxygen 24 hours after installation of NIV. There was no significant difference in the other clinical characteristics of the patients.

Keywords: bronchiolitis viral; noninvasive ventilation; pediatrics. 


\section{INTRODUÇÃO}

A bronquiolite viral aguda (BVA) é a doença respiratória mais comum no primeiro ano da infância. O principal agente associado a esta condição clínica é o vírus sincicial respiratório (VSR), cujo ciclo de vida segue picos sazonais ${ }^{1,2}$. No Rio Grande do Sul, em um estudo de caso-controle, das 5.304 crianças acompanhadas 113 (2,1\%) foram hospitalizadas por BVA no período pós-natal. Outros estudos nesta mesma região do país investigaram a incidência dos vírus na BVA demonstrando que há um predomínio do VSR, variando de $67,8 \%$ a $86,36 \%$ nas amostras estudadas ${ }^{3,4}$.

A BVA constitui-se em um problema de saúde pública, sendo um dos principais motivos de consulta em unidades de emergências pediátricas e uma das maiores causas de hospitalização nos meses de inverno ${ }^{5}$. Nos Estados Unidos ocorrem cerca 22,8 visitas à emergência devido ao VSR para cada 1.000 lactentes, sendo que 29\% necessitaram de internação, o que representa um gasto anual de 50,5 milhões de dólares e com as internações de 650 milhões de dólares ${ }^{6}$.

As alterações na função pulmonar que levam à dificuldade respiratória na BVA são, principalmente, relacionadas a alterações obstrutivas das pequenas vias aéreas (bronquíolos), devido à inflamação aguda, edema e necrose das células epiteliais que revestem as pequenas vias aéreas, produção aumentada de muco e broncoespasmo. A obstrução acarreta alterações nas relações entre ventilação e perfusão e levam à hipoventilação alveolar com hipoxemia, retenção de dióxido de carbono $\left(\mathrm{CO}_{2}\right)$ e acidose respiratória. O quadro obstrutivo leva a um aumento do volume residual e da capacidade residual funcional, resultando em um aumento no trabalho respiratório ${ }^{7,8}$.

Crianças menores de seis meses de idade estão mais predispostas a complicações relacionadas à BVA. Em sua forma mais grave, acompanhada de insuficiência respiratória aguda, com necessidade de ventilação mecânica invasiva (VMI), a BVA pode representar uma importante causa de morbimortalidade. Cerca de 2-6\% dos casos necessitam ser admitidos em Unidade de Terapia Intensiva (UTI) acarretando um maior tempo de internação hospitalar e um aumento substancial dos custos com a internação $0^{8,9,10,11}$.

A ventilação mecânica não invasiva (VMNI) é o fornecimento de suporte ventilatório sem a necessidade de uma via aérea invasiva. Dois tipos de VMNI são comumente usados: CPAP (continuous positive airway pressure) e Bilevel (bilevel positive airway pressure). Na modalidade CPAP uma pressão contínua é fornecida às vias aéreas ao longo de todo o ciclo respiratório proporcionando, dessa forma, aumento na oxigenação, diminuição dos níveis de $\mathrm{CO}_{2}$, redução do trabalho respiratório, diminuição da fadiga dos músculos inspiratórios e prevenção do colapso alveolar devido à pressão contínua de distensão que fornece ${ }^{9,12}$. O modo Bilevel fornece dois níveis de pressão, uma pressão inspiratória (IPAP), que promove auxílio aos músculos respiratórios fadigados e uma pressão expiratória (EPAP), que auxilia na diminuição da hiperinsuflação dinâmica, na permeabilidade das vias aéreas e na diminuição do trabalho respiratório ${ }^{13,14}$.

Estudo desenvolvido por Javouhey et al. ${ }^{9}$, demonstrou que uma estratégia baseada na utilização da ventilação mecânica não invasiva (VMNI) como suporte primário da ventilação em lactentes com bronquiolite grave foi associada com uma menor taxa de pneumonia associada à ventilação, menor duração de oxigenoterapia e com uma tendência de redução no tempo de internação hospitalar. Um estudo de coorte retrospectivo demonstrou que após a implementação do CPAP nasal como primeira estratégia de suporte ventilatório na BVA houve uma redução do custo anual de internação de 715.000 euros $^{15}$.

Um estudo realizado por Milési et al. ${ }^{16}$ comparou o uso do $\mathrm{CPAP}$ à $6 \mathrm{cmH}_{2} \mathrm{O}$ com oxigenoterapia convencional em crianças de até seis meses de idade, internadas em UTI pediátrica com diagnóstico clínico de BVA. Os pacientes que utilizaram CPAP obtiveram uma redução mais rápida do trabalho respiratório. Além disso, a redução do escore de dificuldade respiratória seis horas após o inicio do uso do CPAP foi proporcional à gravidade do paciente, sugerindo a importânica do início rápido do CPAP nas formas mais graves da doença.

Combret et al. ${ }^{17}$ realizaram uma revisão sistemática com 14 estudos recentes incluídos e concluíram que apesar dos resultados promissores em relação ao uso da VMNI na redução da disfunção respiratória na BVA, faltam estudos robustos que confirmem estes resultados. Adicionalmente, nenhum dos estudos incluídos nesta revisão foi realizado em unidade de emerêngia pediátrica.

Apesar dos benefícios já relatados por estudos anteriores do uso da VMNI em crianças com BVA, não há estudos que avaliaram a utilização desta terapêutica na prática clínica de unidades de emergência pediátrica. Portanto, o objetivo do presente estudo foi avaliar os efeitos da VMNI nas primeiras 24 horas de internação em uma emergência pediátrica do sul do país nas variáveis clínicas de lactentes internados com diagnóstico de BVA. Além disso, verificar o tempo de internação hospitalar, a necessidade de oxigenoterapia e os desfechos pós emergência como, internação em UTI ou alta hospitalar.

\section{MATERIAIS E MÉTODOS}

Este estudo foi aprovado pelo Comitê de Alinhamento em Pesquisa do Hospital da Criança Santo Antônio (HCSA) 
e posteriormente pelo Comitê de Ética da Irmandade Santa Casa de Misericórdia de Porto Alegre (ISCMPA) sob o parecer 829.761.

Trata-se de um estudo de coorte retrospectivo, realizado com prontuários médicos de crianças internadas com diagnóstico clínico de BVA que fizeram uso de VMNI na emergência do Hospital da Criança Santo Antônio (HCSA) nos períodos de maio a setembro de 2013 e 2014, caracterizando dois invernos consecutivos, constituindo uma amostra de conveniência. Todas as crianças que internaram na emergência pediátrica com diagnóstico de BVA e fizeram uso da VMNI neste período foram incluídas no estudo.

A busca dos prontuários dos pacientes pediátricos internados nas emergências do Sistema Único de Saúde (SUS) e convênios/particular foi realizada através do programa de gestão em saúde Tasy ${ }^{\circledR}$, utilizando-se a CID J21 (Bronquiolite Aguda), CID J21.0 (Bronquiolite Aguda devida a vírus sincicial respiratório) e CID J21.8 (Bronquiolite Aguda devida a outros microorganismos específicos).

Os dados foram transcritos em uma ficha de avaliação previamente elaborada pelos pesquisadores contendo dados demográficos, características clínicas do paciente como frequência cardíaca $(F C)$, frequência respiratória $(\mathrm{FR})$, saturação periférica de oxigênio $\left(\mathrm{SpO}_{2}\right)$, tipo de vírus, duração da utilização da VMNI (horas), duração da utilização de oxigênio $\left(\mathrm{O}_{2}\right)$ suplementar (dias), tempo de internação hospitalar (dias) e demais desfechos (internação em UTI pediátrica, alta hospitalar ou óbito).

Os dados foram inseridos em uma planilha do Microsoft Excel 2010 para Windows ${ }^{\circledR}$ e analisados através do programa Statistical Package for Social ${ }^{\circledR}$ (SPSS) versão 22.0. As variáveis quantitativas foram expressas em média e desvio-padrão e mediana e intervalo interquartílico e as variáveis qualitativas através de frequência absoluta e percentual. Para as variáveis $\mathrm{O}_{2}, \mathrm{SpO}_{2}$, FC e FR a comparação entre cada período de tempo (seis, 12 e 24 horas) em relação ao momento zero (instalação da VMNI) foi realizada através dos testes não paramétricos Friedman e Wilcoxon. As comparações entre o grupo que utilizou VMI com o grupo que utilizou somente VMNI foi realizada através do teste não paramétrico Mann-Whitney para as variáveis $\mathrm{O}_{2}, \mathrm{SpO}_{2}$ e tempo total de internação hospitalar e o teste para variáveis independentes ANOVA para as variáveis FR e FC. Considerou-se significância estatística o valor de $p$ menor que 0,05.

\section{RESULTADOS}

Durante dois invernos consecutivos 1216 prontuários foram registrados com o CID da BVA. Em uma revisão inicial, 40 prontuários foram excluídos por estarem duplicados ou não disponíveis para análise. No acesso na íntegra aos prontuários, 261 foram excluídos por tratar-se de outro diagnóstico médico que não BVA ou por não haver um diagnóstico definido no momento da alta hospitalar. Onze foram excluídos devido à transferência dos pacientes para UTIs de outros hospitais. Um total de 904 prontuários foram incluídos para análise da utilização da VMNI na emergência pediátrica, bem como para análise dos desfechos clínicos dos pacientes. A Figura 1 apresenta o fluxograma do estudo.
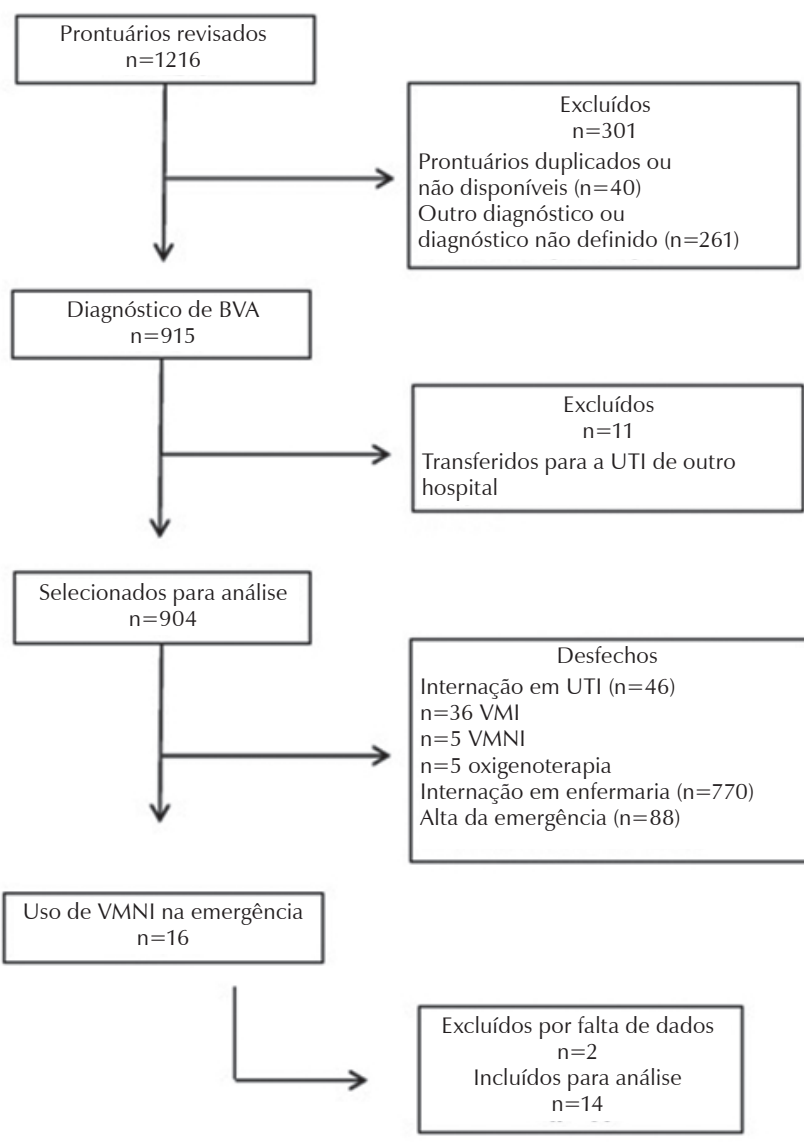

Figura 1. Fluxograma do processo de seleção da amostra e desfechos.

Ao analisar os desfechos dos 904 pacientes internados com BVA, 46 pacientes necessitaram de internação em UTI pediátrica, somando os 11 pacientes que foram transferidos para UTIs de outros hospitais esse número sobe para 57. A maioria dos pacientes $(n=770)$ foi transferida para a enfermaria com posterior alta hospitalar e 88 pacientes tiveram alta da emergência.

No total, 14 crianças fizeram parte da amostra, sendo o primeiro episódio de BVA em todos os pacientes. O tipo de vírus mais prevalente na amostra $(92,8 \%)$ foi o VSR. Duas crianças foram perdidas na análise dos dados, pois as informações de prontuário estavam incompletas. A Tabela 1 apresenta as características da amostra. 
Tabela 1. Características da amostra estudada $(n=14)$.

\begin{tabular}{lc}
\hline \multicolumn{1}{c}{ Variáveis } & $1,5(1,0-2,75)^{*}$ \\
\hline Idade (meses) & $11,93 \pm 5,18$ \\
Internação hospitalar (dias) & $5(35,7 \%)$ \\
Internação em UTI & $8,80 \pm 4,71$ \\
Duração da internação em UTI (dias) & $4(28,6 \%)$ \\
Necessidade de VMI & $10 \pm 1,83$ \\
Duração da utilização da VMI (dias) & $48,00(9,50-96,00)^{*}$ \\
Duração da utilização da VMNI (horas) & $12(85,7 \%)$ \\
Modo CPAP & $2(14,3 \%)$ \\
Modo Bilevel & $9,50 \pm 3,20$ \\
Duração da utilização de $\mathrm{O}_{2}$ (dias) & \\
Raio X de tórax & $12(85,7 \%)$ \\
$\quad$ Atelectasias & $5(35,7 \%)$ \\
\hline Hiperinsuflação &
\end{tabular}

Valores expressos em média e desvio-padrão (DP), *mediana e intervalo interquartílico (P25-75), número (n) e porcentagem (\%). UTI: unidade de terapia intensiva; VMI: ventilação mecânica invasiva; VMNI: ventilação mecânica não invasiva; CPAP: continuous positive airway pressure; Bilevel: bilevel positive airway pressure.

Cinco pacientes apresentaram algum tipo de comorbidade associada, sendo a prematuridade a mais prevalente $(21,4 \%)$, seguida da Sífilis $(14,2 \%)$. A interface utilizada por todos os pacientes durante a VMNI foi a pronga nasal. O modo ventilatório não invasivo predominantemente utilizado foi o CPAP, onde 12 pacientes fizeram uso desse modo ventilatório com uma pressão média no momento da instalação de $7,36 \pm 0,74 \mathrm{cmH}_{2} \mathrm{O}$. A fisioterapia respiratória foi realizada por todos os pacientes no mínimo três vezes ao dia conforme prescrição médica do serviço de emergência.

Quando avaliados os sinais vitais FR $(p=0,374)$, FC $(p=0,966)$, temperatura corporal $(p=0,249)$ e $\mathrm{SpO}_{2}$ $(p=0,175)$, não foram encontradas diferenças significativas entre os valores obtidos no momento da instalação da VMNI em relação à seis, 12 e 24 horas. Já, quando avaliada a utilização de $\mathrm{O}_{2}$ suplementar, houve diferença significativa entre o momento da instalação e após $24 \mathrm{~h}(p=0,024)$ nos 10 pacientes que utilizaram VMNI e não evoluiram para VMI (Figura 2).
Cinco pacientes $(35,7 \%)$ internaram em UTI pediátrica sendo que foi necessária a instituição da VMI em quatro pacientes $(28,6 \%)$. Os sinais que precederam a instituição da VMI nesses quatro pacientes foram: aumento de trabalho ventilatório; associação de aumento de trabalho ventilatório, dessaturação e palidez; associação de aumento de trabalho ventilatório, dessaturação e agitação e associação de aumento de trabalho ventilatório e tiragens.

Os pacientes que utilizaram VMI permaneceram menos de 12 horas com suporte ventilatório não invasivo, por este motivo, a comparação dos sinais vitais entre os pacientes que utilizaram somente $\mathrm{VMNI}$ com os pacientes que utilizaram VMI foi realizada no momento da instalação da VMNI e após seis horas desta. A FC foi o sinal vital que diferiu significativamente tanto no momento da instalação da VMNI $(p=0,002)$ como em relação a seis horas após a instalação $(p=0,041)$ podendo dessa forma, ter sido um dos fatores relacionados a falha da VMNI. A FR e a necessidade de $\mathrm{O}_{2}$ suplementar, apesar de apresentarem redução entre o momento da instalação da VMNI em relação a seis horas, não houve diferença estatisticamente significativa. De igual forma, não houve diferença na $\mathrm{SpO}_{2}$ nos dois momentos avaliados (Tabela 2).

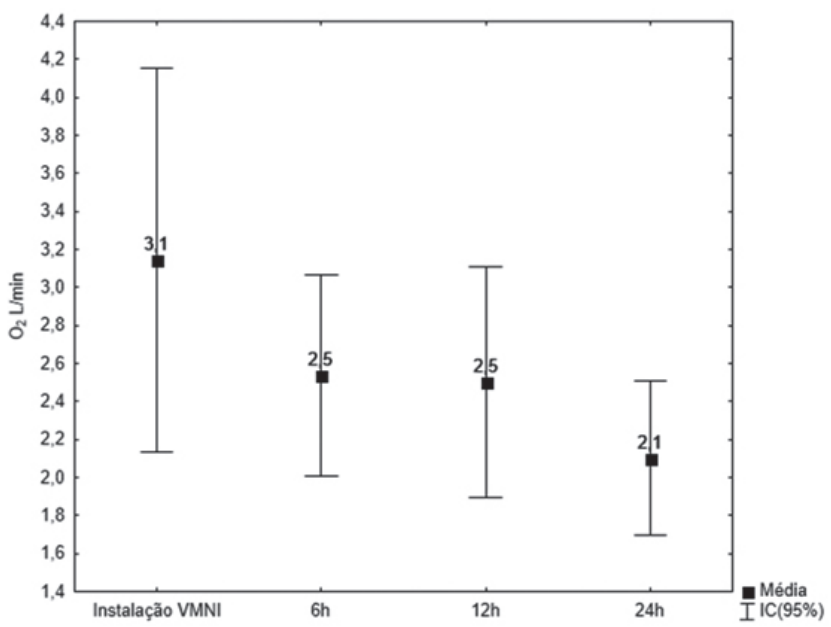

Figura 2. Utilização de oxigênio suplementar.

Tabela 2. Comparação entre os pacientes que utilizaram VMI e os que utilizaram somente VMNI.

\begin{tabular}{|c|c|c|c|c|c|c|}
\hline \multirow[b]{2}{*}{ Variável } & \multicolumn{3}{|c|}{ Momento da instalação da VMNI } & \multicolumn{3}{|c|}{6 horas } \\
\hline & $\begin{array}{c}\text { Grupo VMI* } \\
\qquad(n=4)\end{array}$ & $\begin{array}{c}\text { Grupo VMNI } \\
(\mathrm{n}=10)\end{array}$ & $p$ & $\begin{array}{c}\text { Grupo VMI* } \\
\quad(n=4)\end{array}$ & $\begin{array}{c}\text { Grupo VMNI } \\
(\mathrm{n}=10)\end{array}$ & $p$ \\
\hline FC & $174,75 \pm 12,28$ & $145,20 \pm 13,05$ & 0,002 & $173,00 \pm 7$ & $146,25 \pm 18,45$ & 0,041 \\
\hline FR & $62,33 \pm 6,66$ & $53,40 \pm 11,82$ & 0,246 & $45,33 \pm 15,04$ & $47,00 \pm 5,63$ & 0,867 \\
\hline $\mathrm{O}_{2}$ & $3,50 \pm 3,11$ & $3,00 \pm 1,05$ & 0,733 & $2,00 \pm 1,00$ & $2,70 \pm 0,82$ & 0,287 \\
\hline $\mathrm{SpO}_{2}$ & $98,75 \pm 1,50$ & $100,00 \pm 0,00$ & 1,000 & $98,80 \pm 1,40$ & $99,78 \pm 0,44$ & 0,600 \\
\hline
\end{tabular}

*Pacientes que falharam na VMNI e necessitaram de suporte ventilatório invasivo.

$\mathrm{FC}$ : frequência cardíaca (bpm); FR: frequência respiratória $(\mathrm{rpm}) ; \mathrm{O}_{2}$ : oxigênio $(\mathrm{L} / \mathrm{min}) ; \mathrm{SpO}_{2}$ : saturação periférica de oxigênico (\%);

VMI: ventilação mecânica invasiva; VMNI: ventilação mecânica não invasiva; 6 horas: seis horas após a instalação da VMNI. 


\section{DISCUSSÃO}

$\mathrm{Na}$ literatura, está bem estabelecido que em criança menores, a gravidade clínica e o impacto da BVA pelo VSR são maiores. A idade média das crianças incluídas no nosso estudo foi de 1,88 $\pm 1,25$ meses. Estudo realizado na Espanha avaliou 229 pacientes internados em UTI pediátrica por BVA e constatou que a idade foi inversamente proporcional ao tempo necessário de suporte ventilatório e de dias de internação em UTI 18,19,20. Um estudo realizado nos Estados Unidos apontou que de 1,1 milhão de crianças menores de dois anos internadas por VSR em um período de oito anos, a maior porcentagem das internações ocorreu na faixa etária entre três e seis meses ${ }^{21}$.

O VSR foi o vírus mais prevalente na amostra estudada $(92,8 \%)$ durante dois invernos consecutivos. Estudos que compararam grupos de pacientes com BVA devido ao VRS com pacientes com BVA devido a outros vírus, encontraram maior tempo de internação hospitalar, maior escore clínico de gravidade, maior risco de internação em UTI, maior percentual de necessidade de suporte ventilatório e maior necessidade de oxigenoterapia no grupo VSR positivo, sugerindo que a BVA causada por esse vírus é uma doença mais grave do que aquela causada por outros vírus ${ }^{22,23}$.

As alterações radiográficas mais prevalentes foram as atelectasias em 12 pacientes e a hiperinsuflação pulmonar em cinco, sendo que oito pacientes apresentaram mais de uma alteração radiográfica. No estudo realizado por Hervás et al. ${ }^{23}$ a presença de atelectasias e consolidações pulmonares apresentaram associação com aumento na necessidade de internação em UTI, necessidade de oxigenoterapia e duração da internação hospitalar, tanto em crianças com BVA devido ao VRS como devido a outros vírus.

Em relação a comorbidades, três $(21,4 \%)$ pacientes eram prematuros. Os recém-nascidos prematuros, devido à imaturidade pulmonar, são mais suscetíveis ao desconforto e à insuficiência respiratória ${ }^{24}$. Um estudo realizado na França comparou a taxa de hospitalização por BVA de crianças prematuras (idade gestacional $<33$ semanas) com crianças nascidas a termo e encontrou uma taxa de hospitalização significativamente maior no primeiro grupo $(14,1 \%$ versus $2,0 \% ; p<0,0001)$. Além disso, o grupo de prematuros apresentou um risco sete vezes maior de internação comparado ao grupo de nascidos a termo, todos com idade inferior a seis meses ${ }^{25}$.

A pronga nasal foi a interface utilizada pelos 14 pacientes. A aplicação da VMNI pode ser realizada por intermédio de diferentes tipos de interfaces, sendo sua escolha um passo primordial no sucesso da $\mathrm{VMNI}$, pois a adaptação do doente dependerá das suas características ${ }^{26}$. O tamanho da interface e o posicionamento correto do paciente são importantes para que a ventilação seja eficaz ${ }^{12}$. Em relação ao modo ventilatório não invasivo, 12 pacientes fizeram uso do CPAP com uma pressão média no momento da instalação de sete $\mathrm{cmH}_{2} \mathrm{O}$. Estudos recentes apontam que em crianças com insuficiência respiratória por BVA níveis de CPAP nasal de sete $\mathrm{cmH}_{2} \mathrm{O}$ estão associados a menor sobrecarga dos músculos respiratórios e melhora do padrão respiratório, bem como uma evolução clínica favorável em curto prazo $^{27}$, requerendo, dessa forma, menor duração de suporte ventilatório ${ }^{28}$.

Quatro pacientes $(28,6 \%)$ falharam na VMNI, foram internados em UTI pediátrica e utilizaram VMI. Esses pacientes, comparativamente aos que tiveram sucesso na utilização da VMNI e tiveram desfecho de alta hospitalar, apresentaram diferença significativa em relação à FC no momento da instalação da VMNI e em relação a seis horas, podendo este ser um fator associado à falha da VMNI. No estudo de Colunga et al..$^{29}$ que teve como objetivo identificar fatores preditivos de falha na VMNI, o grupo de pacientes que necessitou de $\mathrm{VMI}$ apresentou nenor idade e peso, maior escore clínico de gravidade, maior FC e FR após 24 horas da instalação da VMNI em relação aos pacientes que obtiveram sucesso na VMNI. Além disso, três fatores de risco independentes para a falha da VMNI foram identificados: insuficiência respiratória hipoxêmica, elevado escore clínico de gravidade e diminuição não expressiva da FR depois de uma a seis horas de VMNI, achado este corroborando com os nossos.

Em relação ao total de internações em UTI pediátrica, $6,2 \%$ dos pacientes tiveram essa necessidade, sendo que 3,9\% fizeram uso de VMI durante a internação na UTI do hospital onde o estudo foi realizado, dados esses que corroboram com os achados da literatura. A mediana de utilização da VMNI foi de 48 horas, variando entre duas e 120 horas. O tempo médio de internação hospitalar dos pacientes que utilizaram somente $\mathrm{VMNI}$ foi significativamente menor em relação aos pacientes que necessitaram suporte ventilatório invasivo. Resultados semelhantes foram encontrados por Abadesso et al. ${ }^{30}$ que avaliaram a eficácia clínica da VMNI em evitar a intubação orotraqueal em crianças com insuficiência respiratória aguda cujo principal diagnóstico foi a BVA. A duração média de utilização de VMNI foi de 48,3 \pm 39 horas, variando entre uma e 209 horas. Neste mesmo estudo, o tempo médio de internação hospitalar no grupo que utilizou somente VMNI foi de 8.4 48.8 dias, já no grupo que falhou na VMNI e necessitou de VMI o tempo médio de internação hospitalar foi de $14,5 \pm 10,2$ dias $(p<0,003)$. 
Essouri et al. ${ }^{15}$ realizaram um estudo de coorte retrospectivo para avaliar as consequências econômicas da implementação de CPAP nasal como primeiro suporte ventilatório em crianças com BVA em um hospital francês. Dividiram os pacientes em dois grupos: grupo 1 antes da implementação da VMNI (1996-2000) e grupo 2 após a implementação (2006-2010). O grupo 2 apresentou diminuição significativa do tempo de suporte ventilatório em relação ao grupo 1, menor tempo de internação em UTI e menor tempo de internação hospitalar. O custo médio das internações em UTI pediátrica relacionadas com BVA foi reduzido significativamente de 17.451 para 11.205 euros $(p<0,001)$. Além disso, a utilização de CPAP nasal foi associada com uma baixa taxa de falha do tratamento entre 15 e $3 \%$ no ano mais recente.

Nosso estudo apresentou algumas limitações tais como pequeno tamanho amostral, falta de dados gasométricos dos pacientes, assim como a falta de um grupo controle que não utilizou VMNI para comparação das variáveis clínicas. Além disso, por se tratar de dados de prontuários pode haver um viés de aferição nos dados coletados.

Concluimos que, apesar de não poder mensurar a diferença na $\mathrm{PaO}_{2}$ na amostra estudada, houve redução significativa da necessidade de $\mathrm{O}_{2}$ suplementar após 24 horas da instalação da VMNI. Não foram observadas diferenças significativas nas demais características clínicas dos pacientes. Devido a excasses de estudos realizados em unidades de emergência pediátrica com a utilização de VMNI, torna-se importante a realização de estudos experimentais a fim de identificar os reais benefícios da VMNI na população pediátrica com diagnóstico clínico de BVA.

\section{REFERÊNCIAS}

1. Pupin MK, Riccetto AGL, Ribeiro JD, Baracat ECE. Comparação dos efeitos de duas técnicas fisioterapêuticas respiratórias em parâmetros cardiorrespiratórios de lactentes com bronquiolite viral aguda. J Bras Pneumol. 2009;35(9):860-7. https://doi.org/10.1590/ S1806-37132009000900007

2. Gomes ELFD, Postiaux G, Medeiros DRL, Monteiro KKDS, Sampaio LMM, Costa D. Chest physical therapy is effective in reducing the clinical score in bronchiolitis: randomized controlled trial. Rev Bras Fisioter. 2012;16(3):241-7. https://doi.org/10.1590/S141335552012005000018

3. Sudbrack S, Ponzi D, Massuco L, Coral C, Stein PT, Pitrez PMC. Prevalência de vírus respiratórios em lactentes com bronquiolite aguda e sibilância recorrente em uma emergência pediátrica no sul do Brasil. Sci Med. 2007;17(3):124-9.

4. Sparremberger DAH, Luisi F, Azevedo AV, Ribeiro AET, Wiemann AFW, de Conto BF, Munhoz BZ, Morais EFM, Bergmann GA, Maróstica LC, Valiati LS, Spinelli LF, Sussela LAO, Nunes MR, Regert R, Lardi SL, Pinto LA, Stein RT. Características epidemiológicas e influência da coinfecção por vírus respiratórios na gravidade da bronquiolite aguda em lactentes. Sci Med. 2011;21(3):101-6.

5. Luisi F. O papel da fisioterapia respiratória na bronquiolite viral aguda. Sci Med. 2008;18(1):39-44.

6. Leader S, Kohlhase K. Recent trends in severe respiratory syncytial virus (RSV) among US infants, 1997 to 2000. J Pediatr. 2003;143(Suppl 5):S127-32. https://doi.org/10.1067/S00223476(03)00510-9

7. Perrotta C, Ortiz Z, Roque M. Chest physiotherapy for acute bronchiolitis in paediatric patients between 0 and 24 months old. Cochrane Database Syst Rev. 2005;(2):CD004873. https://doi. org/10.1002/14651858.CD004873.pub2

8. American Academy of Pediatrics Subcommittee on Diagnosis and Management of Bronchiolitis. Diagnosis and management of bronchiolitis. Pediatrics. 2006;118(4):1774-93. https://doi. org/10.1542/peds.2006-2223

9. Javouhey E, Barats A, Richard N, Stamm D, Floret D. Non-invasive ventilation as primary ventilatory support for infants with severe bronchiolitis. Intensive Care Med. 2008;34(9):1608-14. https://doi. org/10.1007/s00134-008-1150-4

10. Nizarali Z, Cabral M, Silvestre C, Abadesso C, Nunes P, Loureiro $H$, Almeida $H$. Ventilação não invasiva na insuficiência respiratória aguda na bronquiolite por vírus sincicial respiratório. Rev Bras Ter Intensiva. 2012;24(4):375-80. https://doi.org/10.1590/S0103507X2012000400014

11. Cambonie G, Milési C, Jaber S, Amsallem F, Barbotte E, Picaud JC, Matecki S. Nasal continuous positive airway pressure decreases respiratory muscles overload in young infants with severe acute viral bronchiolitis. Intensive Care Med. 2008;34(10):1865-72. https:// doi.org/10.1007/s00134-008-1201-x

12. Loh LE, Chan $\mathrm{YH}$, Chan I. Noninvasive ventilation in children: a review. J Pediatr (Rio J). 2007;83(2 Suppl):S91-9. https://doi. org/10.2223/JPED.1613

13. Najaf-Zadeh A, Leclerc F. Noninvasive positive pressure ventilation for acute respiratory failure in children: a concise review. Ann Intensive Care. 2011;1(1):15. https://doi.org/10.1186/2110-5820-1-15

14. Calderini E, Chidini G, Pelosi P. What are the current indications for noninvasive ventilation in children? Curr Opin Anaesthesiol. 2010;23(3):368-74. https://doi.org/10.1097/ ACO.0b013e328339507b

15. Essouri S, Laurent M, Chevret L, Durand P, Ecochard E, Gajdos V, Devictor $\mathrm{D}$, Tissières $\mathrm{P}$. Improved clinical and economic outcomes in severe bronchiolitis with pre-emptive nCPAP ventilatory strategy. Intensive Care Med. 2014;40(1):84-91. https://doi.org/10.1007/ s00134-013-3129-z

16. Milési $C$, Matecki $S$, Jaber $S$, Mura $T$, Jacquot $A$, Pidoux $O$, Chautemps N, Novais AR, Combes C, Picaud JC, Cambonie G. 6 $\mathrm{cmH} 2 \mathrm{O}$ continuous positive airway pressure versus conventional oxygen therapy in severe viral bronchiolitis: a randomized trial. Pediatr Pulmonol. 2013;48(1):45-51. https://doi.org/10.1002/ ppul.22533

17. Cambret Y, Prieur G, Le Roux P, Médrinal C. Non-invasive ventilation improves respiratory distress in children with acute viral bronchiolitis: a systematic review. Minerva Anestesiol. 2017;83(6): 624-37. 
18. Alvarez AE, Marson FAL, Bertuzzo CS, Arns CW, Ribeiro JD. Epidemiological and genetic characteristics associated with the severity of acute viral bronchiolitis by respiratory syncytial virus. J Pediatr (Rio J). 2013;89(6):531-43. https://doi.org/10.1016/j. jped.2013.02.022

19. O-oro G, Suárez EP, Bouzas MII, Serrano A, De Azagra AM, GarcíaTeresa MA, Flores JC. Bronquiolitis grave. Cambios epidemiológicos y de soporte respiratório. An Pediatr (Barc). 2011;74(6):371-6. https://doi.org/10.1016/j.anpedi.2011.01.012

20. Stockman LJ, Curns AT, Anderson LJ, Fisher-Langley G. Respiratory syncytial virus-associated hospitalizations among infants and young children in the United States, 1997-2006. Pediatr Infect Dis J. 2012;31(1):5-9. https://doi.org/10.1097/INF.0b013e31822e68e6

21. Fryzek JP, Martone WJ, Groothuis JR. Trends in chronologic age and infant respiratory syncytial virus hospitalization: an 8-year cohort study. Adv Ther. 2011;28(3):195-201. https://doi.org/10.1007/ s12325-010-0106-6

22. Rödl S, Resch B, Hofer N, Marschitz I, Madler G, Eber E, Zobel G. Prospective evaluation of clinical scoring systems in infants with bronchiolitis admitted to the intensive care unit. Eur J Clin Microbiol Infect Dis. 2012;31(10):2667-72. https://doi.org/10.1007/s10096012-1612-z

23. Hervás D, Reina J, Ya-es A, del Valle JM, Figuerola J, Hervás JA. Epidemiology of hospitalization for acute bronchiolitis in children: differences between RSV and non-RSV bronchiolitis. Eur J Clin Microbiol Infect Dis. 2012;31(8):1975-81. https://doi.org/10.1007/ s10096-011-1529-y

24. Gonzaga AT, Figueira BBD, Sousa JMA, Carvalho WB. Tempo de ventilação mecânica e desenvolvimento de displasia bronco- pulmonar. Rev Assoc Med Bras. 2007;53(1):64-7. https://doi. org/10.1590/S0104-42302007000100022

25. Gouyon JB, Rozé JC, Guillermet-Fromentin C, Glorieux I, Adamon L, Di Maio M, Miloradovich T, Anghelescu D, Pinguier D, Escande B, Elleau C. Hospitalizations for respiratory syncytial virus bronchiolitis in preterm infants at $<33$ weeks gestation without bronchopulmonary dysplasia: the CASTOR study. Epidemiol Infect. 2013;141(4): 816-26. https://doi.org/10.1017/S0950268812001069

26. Nunes P, Abadesso C, Almeida E, Silvestre C, Loureiro H, Almeida H. Ventilação não invasiva numa unidade de cuidados intensivos pediátricos. Acta Med Port. 2010;23(3):399-404.

27. Essouri S, Durand P, Chevret L, Balu L, Devictor D, Faroux B, Tissières, P. Optimal level of nasal continuous positive airway pressure in severe viral bronchiolitis. Intensive Care Med. 2011;37(12): 2002-7. https://doi.org/10.1007/s00134-011-2372-4

28. Borckink I, Essouri S, Laurent M, Albers MJIJ, Burgerhof JGM, Tissières $P$, Kneyber MC. Infants with severe respiratory syncytial virus needed less ventilator time with nasal continuous airways pressure then invasive mechanical ventilation. Acta Paediatr. 2014;103(1):81-5. https://doi.org/10.1111/apa.12428

29. Mayordomo-Colunga J, Medina A, Rey C, Díaz JJ, Concha A, Los $\operatorname{Arcos} M$, Menéndez S. Predictive factors of non invasive ventilation failure in critically ill children: a prospective epidemiological study. Intensive Care Med. 2009;35(3):527-36. https://doi.org/10.1007/ s00134-008-1346-7

30. Abadesso C, Nunes P, Silvestre C, Matias E, Loureiro H, Almeida $\mathrm{H}$. Non-invasive ventilation in acute respiratory failure in children. Pediatr Rep. 2012;4(2):e16. https://doi.org/10.4081/pr. 2012.e16 